\title{
ON THE DEVELOPMENT OF AN OPEN PLATFORM FOR M-GOVERNMENT SERVICES
}

\author{
Helena Rodrigues, César Ariza and Jason Pascoe \\ Dep. Sistemas de Informação, Universidade do Minho, 4800-058 Guimarães, Portugal
}

\begin{abstract}
Citizens and local government services are often not as well connected as they ideally should be. Services may not be well advertised or may simply be cumbersome or time-consuming to access. In this paper we present our ongoing work in investigating hew to better connect the citizen with their local government with the support of context-aware applications. We describe a set of user requirements for m-government services and open service-oriented platforms. In particular, we analyze the requirements and present the research issues on a context modeling component for supporting context-aware service discovery. Our motivation to develop it is driven by the need to provide appropriate local goveınment services in an easily accessible manner whenever and wherever they may be needed.
\end{abstract}

Keywords: m-government servics, m-government user recuirements, context-awareness, context-aware service discovery.

\section{INTRODUCTION}

Mobile technologies provide an inıportant alternative channel for the interaction between authorities and citizens. This channel is very important as such for two reasons. Firstly, they are accessible to many more people than traditional desktop computing. The penetration rate for mobile phones in most European countries is already above $75 \%$, allowing services to be reached by a much larger population and helping to break the digital divide. Secondly, these channels have the characteristics of allowing nearly immediate communication, thus supporting time-constrained notifications that would not be possible by other channels, and allowing applications such as alerts, traffic reports, complaints and promotion of events to be implemented. 
Our work is part of a pan-European project called USE-ME.GOV (USEME.GOV consortium, 2002) whose general objective is to promote a better connection between citizens and their local government services through mobile technology. The project vision is centered on the provision of appropriate services (i.e context-dependent services) to the citizen directly in the time and place in which they are needed and/or useful. Contextawareness (Schilit et al., 1994), the ability of a system to sense, react, and adapt to its environment of use, can be used to discover, filter and prioritize the set of services appropriate for a citizen's current context. For example, considering a service to report anomalies to public authorities, when located in a park it is quite probable that the citizen's complaint will be directed to the parks and gardens authority, a nearby a water main may also present a possible source of concern in the event of a burst pipe, or a history of graffiti in the area may prompt the system to select the graffiti correction department, and there is also a good chance that the citizen's complaint may be the same as other recently reported complaints in the locality.

There have been recently several approaches to m-government (see section 2). The reviewed projects mainly present the lack of support from an open and interoperable architecture for the sharing of external content providers, mobile operator interfaces, context information and third-party services. In this paper we present our work that consisted in the analysis of a set of user requirements for m-government services and the derivation on a set of systems requirements for an open architecture for the development of context-aware applications. We mairly focus the requirements for a context modeling component as the support for m-government context-aware applications.

The paper is organized as foilowing. In section 2 we review existing mgovernment platforms. In section 3 we describe the set of user requirements for an open service platform for m-government services. In section 4 we desctibe the logical architectural model of USE-ME.GOV platform and describe, particulaily, the requirements on service repository and service discovery components, as well as requirements on context modeling component. In section 5 we present our work on a context modeling component design and, finally, in section 6 we present our conclusions and future work.

\section{REVIEW OF M-GOVERNMENT PLATFORMS}

In the last years there have been several approaches to mobile government. Such approaches share common objectives with USEME.GOV, aithough addressing different issues. The mGovernment Service 
initiative in Malta (Government of Malta, 2003) offers e-government services to mobile users using SMS (Short Message System) technology. Services are limited to notification services as the interaction format is mainly text and are not context-based. As far we understood, the components of the technological architecture are mainly the government services and a component that communicates with mobile operator for routing the SMS messages. The main limitations of this architecture is that it does not offer any API (Application Programming Interface) for sharing content between services, for integrating third party service providers or for integrating new mobile applications.

The Visual Admin project initiative (Visual Admin consortium, 2000) offers significant functionality to citizens through an e-government Web Portal. The ubiquitous access they provide is limited to offering a new channel to access the portal (GPRS), giving up all the opportunities offered by the mobile communications technology such as the location services components. Actually, one of the results of the project states that users mentioned that some of the functionalities on the fixed interface should not appear in the mobile one. The technological architecture is mainly based on database and search engine technology, what may compromise scalability and openness.

The Pandora project (Pandora consortium, 2001) presents some similar objectives to the ones of USE-ME.GOV in what concerns the provided functionality to the user, although not considering context-awareness. The project objective is to specify and implement a new Mobile Content Management Platform able to access, manage and deliver wireless multimedia/multilingual infornation from Web pages, local/regional databases, and othe: content sources, exploiting UMTS technology features. At the current stage, the main objective of USE-ME.GOV project is to specify and implement a platform for the deployment of e-government services, evolving from the traditional approaches of Web information filtering to the concept of an open platform that offers sharing of common content and application programming interfaces for the development of new services and mobile applications.

The CENTURi21 project (CENTURi21 consortium, 1999) also presents some similar objectives to the ones of USE-ME.GOV in what concerns the provided functionality to the user, although not considering contextawareness. The results on interoperability and integration of services may be of USE-ME.GOV interest. However, the target context of USE-ME.GOV project is a local context as opposing to a global target context of CENTURi21 project. The late one does not explores the relevance of user context and location to improve both usability and sharing. 
The Mobhaile project (Mobhaile project, 2004) presents a set of very similar functionalities to the one in USE-ME.GOV, including context-aware functionality. Apparently, the supporting platform does not support integration of third-party services and application providers, compromising scalability and openness. Although offering a friendly interface, such as a Map interface, this platform does not apparently offer the advantage of mobile communications technology such as location services.

\section{ANALYSIS OF REQUIREMENTS FOR M- GOVERNMENT SERVICES}

\subsection{Use Case Description}

Functional requirements capture the intended behavior of the system from the users' point of view. This behavior may be expressed as services or functionalities the system is required to perform. In this section, we define a set of use cases built after an analysis of user requirements for $\mathrm{m}$ government services in the context of USE-ME.GOV project. These use cases describe the set of functional requirements of USE-ME.GOV platform. We have defined two orthogonal criteria to breakdown the USE-ME.GOV platform functionalities (see figure 1). The first one, criteria a (left), describes the main functionalities of the system and the second one, criteria $b$ (right), defines the different application domains.
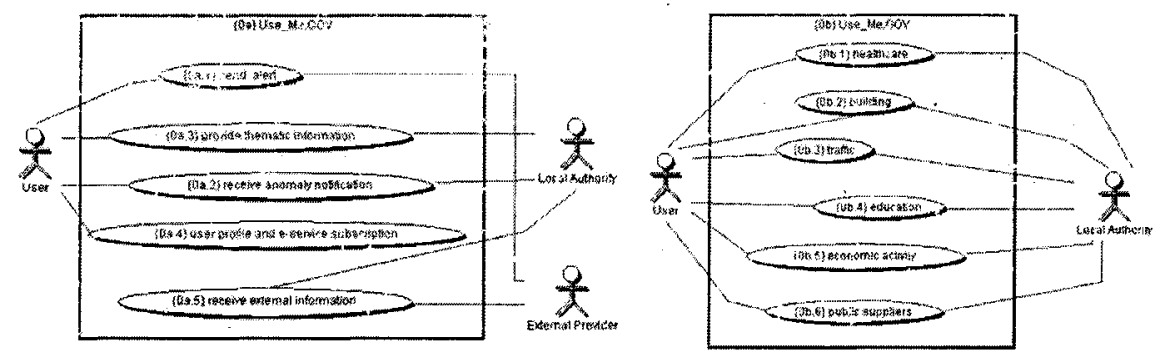

Figure 1. Functionalities of the m-government platform.

The user is the beneficiary of functionality provided by an application that is, to some extent, based on the capabilities of the USE-ME.GOV platform. We assume that the user is a subscriber of a mobile network and carries some communicating device.

The USE-ME.GOV platform assumes a special case of external provider that is the Local Authority. At this stage, we distinguish Local authority 
from other external providers as they are the entities defining and providing the ultimate services to the user and are also a beneficiary of the platform.

The USE-ME.GOV platform assumes that many other entities can contribute to the system by providing general information (geographic information, traffic related information, tourism information ...) and mobile operators network services (the mobile networks themselves).

We are now presenting a discussion on each of the identified functionalities for an m-government platform in which are based our system's requirements.

send alert: This is an asynchronous service and refers to the dissemination of particular domain dynamic information to mobile users informing on specific events and situations or unexpected situations that are happening in the region. Such functionality is particularly interesting for the mobile setting as user context, such as user location, time, activity, preferences, etc, may determine the set of users the alert should be sent and determine the adequate granularity of information. For example, an alert service could be used for warning users in a certain geographic area about a traffic congestion, flood, weather storm, etc. The information associated to the alert should always be up-to-date and match the user-specific request, excluding any extra information or undesired advertisements. For those users that require personalized information, subscription services are necessary.

receive anomaly notification: This is a synchronous service that consists on receiving a report from users informing of some anomaly of a particular domain. Such functionality is particularly interesting for the mobile setting as this service may take into consideration the information on user context, such as location, to characterize the reported situation. For example, a user report, when located in a garden, woula be direci to gardens authority. It would also be helpful to allow the user to navigate to the appropriate level of context information granularity, starting from the highlevel context up to the level of user awareness, in order to get mor: accurate information on user context. For example, if the user chcoses to complaint about the bad state of a flower bed in a garden, the dialogue may provide a set of gardens located in an automatic retrieved wide area location, and ask the use to select the correct one.

provide thematic information: This is a synchronous service that consists on provide information of a particular domain to user on demand. Such functionality is particularly interesting for the mobile setting as this service may take into account the user context information (user's geographic location, time, user's current activity) to select the appropriate information from the distributed information space. For example, if the user requests information on local events or local businesses, for example within the context of industrial or thematic fairs, the service may use his location 
information to select the appropriate services. For those users that require personalized information, subscription services are necessary.

user profile and e-service subscription: The user registers into the system providing his profile and the set of subscribed services. Particularly, for alert functionality, users subscribe required alert services, for example. If users want to receive personalized information via both send alert and provide thematic information they should be able to create a user profile with personal identification and preferences specific to each particular domain service.

receive external information: The USE-ME.GOV platform includes the functionality to receive/request information in a particular domain from/to external content providers. For example, a geographic information system provides landmarks geographic positioning for supporting users localization process, or, a traffic information external provider provides traffic congestion alerts that may support traffic alert services. Contents/events and related information are to be delivered by the system (via send alert and provide thematic information) to the users basing on users' profiles and users' context.

The functionalities we have described exhibit a set of common characteristics. They characterize mainly a spontaneous user interaction with the system, the USE-ME.GOV platform. M-government services should be characterized by a "whenever and wherever" usage, that is, the service must be always available and accessed right at the point in time the user requires its funetionality.

M-government services should be contextualized and should consider the current situation, or context, of the user during the service utilization. The context of a user, or other entity, is deined by several types of context (sometimes knowir at context dimensions), such as time, location, activity and preferences (Dey et al., 1999; Schmidt et al., 1998). Context should be explored by m-government services in the sense that it characterizes the user situation and can be used to interpret user's explicit acts, making communication (between users and services) much more efficient.

M-government services should integrate with different external content providers to collect, aggregate and present useful information to users.

\subsection{The Report Complaint Scenario}

Local governments often have a multitude of disparate internal and external complaint procedures. For example, a pothole in the road may have to be reported directly to the highway maintenance department and then internally passed on to the local road repair team responsible for that section of road. As a citizen it can be rather off-putting to have to locate and 
navigate one's way both to and through the appropriate local government offices (or, in some cases, web sites) in order to report such a complaint. It forms a high barrier-to-use. However, in our solution a citizen is expected to be automatically connected to an appropriate complaint service whilst directly at the scene of the trouble, using their own personal wireless device (such as a PDA, mobile phone, or wearable computer).

\section{SYSTEM REQUIREMENTS FOR M- GOVERNMENT SERVICES}

In this section we analyze the result of transforming the requirements specified in the previous section into a set of system requirements that can affect an appropriate decomposition of the system and the assignment of responsibilities to high-level components. The applied transformational methodology is described in (Machado et al., 2005) and it was applied by a different team and is out of scope of this paper. The derived object model, the USE-ME.GOV platform logic architectural model, is presented in figure 2 . We may identify in figure 2 a Service Repository component (objects $\{O 0 a .1 .5 . d\}$ and $\{00 a .1 .5 . i\})$, a Service Discovery Protocol component (objects $\{00 a .2 .5 . c\}$ and $\{00 a .2 .5 . i\}$ ) and a Context Modeling component (package $\{\mathrm{P} 3\}$.$) . Such types of components are typically found in service-$ oriented architectures for the mobile environment (Satyanarayanan, 2001).

\subsection{Service Repository and Service Discovery Protocol}

Service discovery enables networked entities to discover each other, exchange their functional capabilities, and possibly enter into a relationship. Such component in USE-ME.GOV platform is responsible for discovering, from the known multi-service environment, the appropriate services for accomplish the user request. In order to support efficient service discovery and service aggregation, the service repository component should store the provided services attributes. A proper service description mechanism should be introduced.

We can find in the literature and industrial scene various service-oriented architectures like CORBA (OMG, 2004), UDDI (OASIS, 2003) and Jini (Arnold et al., 1999). These technologies take different approaches in terms of how they support the service discovery process, which result from the various application domains for which they were created or from specific assumptions about their network or computing environments. Particularly, CORBA and UDDI are designed for the Wide Area Network (WAN) setting and Jini for the Local Area Network (LAN) setting. The later is mainly 
dependent of particular LAN technologies such as multicast protocols for dynamic discovery of lookup services. In the WAN setting, CORBA objects are potentially disadvantageous because of the constantly changing environment of the Internet. A major restriction of the distributed objectoriented programming approach is that the interactions among objects are fixed through explicitly coded instructions by the application developer. This implies that it is very difficult to reuse an object in a new application without bringing along all its inherent dependencies on other objects (embedded interface definitions and explicit method calls).

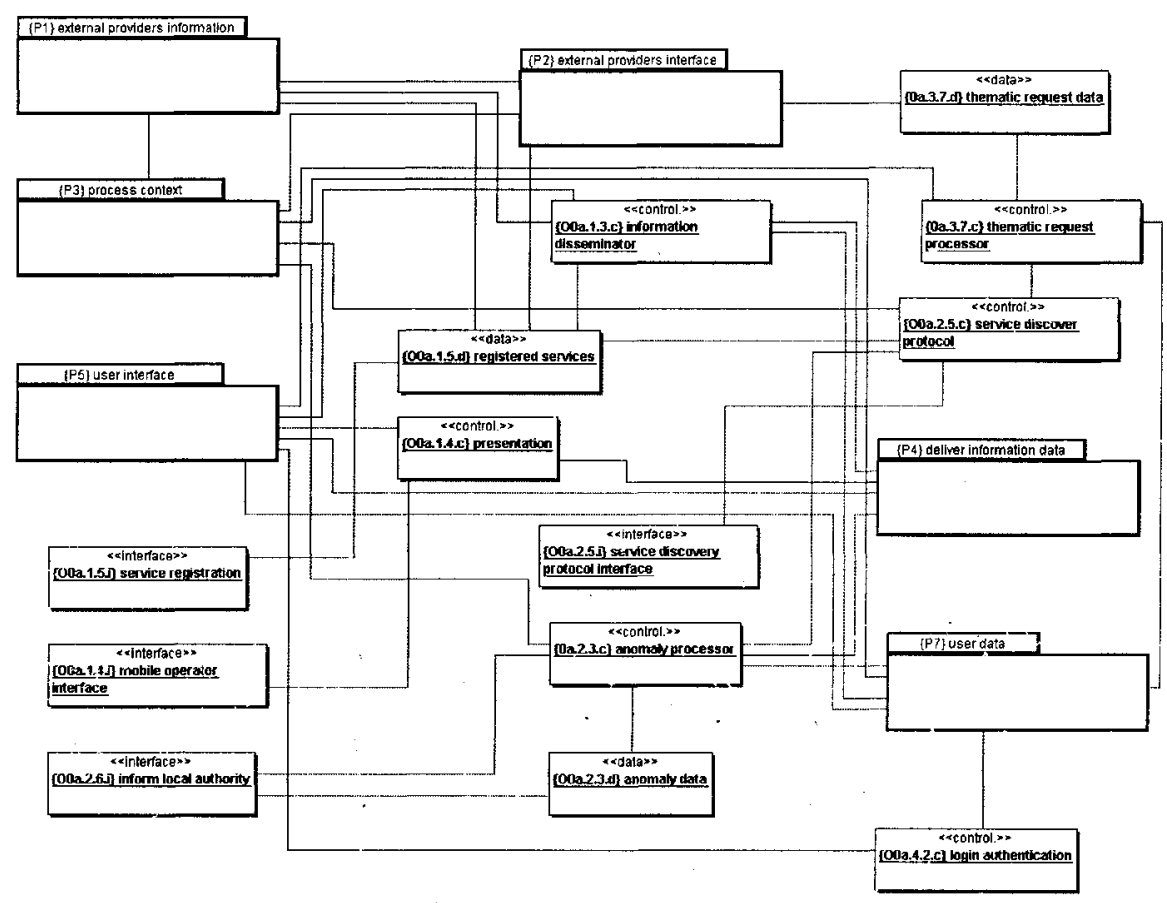

Figure 2. USE-ME.GOV platform logic architectural model

For description purposes, a separate USE-ME.GOV team has designed a meta-protocol of service types based on OWL-S (Bassara et al., 2004). OWL-S is an OWL-based Web service ontology, which supplies Web service providers with a core set of markup language constructs for describing the properties and capabilities of their Web services in unambiguous, computer-interpretable form. OWL-S markup of Web services will facilitate the automation of Web service tasks, including automated Web service discovery, execution, composition and interoperation (World Wide Web consortium, 2004). 


\subsection{Context Modeling Component}

As a result of their mobility, users have a much closer relationship with their surrounding physical environment, and will thus have a much stronger need for information that relates to that environment and their current situation. This need has driven the development of models for supporting the provision of context-aware applications in which information services are associated with particular locations and other context information.

A key research challenge in the development of such systems is how to allow context-aware applications to discover the services they need for the current user situation. Different aspects of context like the position, time, personal preferences or costs should be considered in order to discover a service which matches the user's needs best. Context-aware service discovery is an issue that has been prone to several approaches in recent years, resulting in the development of several standards and technologies. There has been considerable work targeted at supporting location-aware services discovery in mobile computing environments (Cheverst et al., 2000; José et al., 2003; McGrath, 2000), and in context-aware architectures in general (Abowd et al., 1997; Salber et al., 1999). Such systems currently develop individual solutions that only address the requirements of specific application scenarios, are only valid for the specific underlying technology on which they are based or are based on location and context models that are insufficient for associating a service with user situation, mainly because of the different iypes and scales of known location and context information.

Building context-aware services for mobile environinents relies on many different technologies such as context acquisition, context modeling and representation, context reasoning and context-aware service discovery. Mobile informaiion systems maintain many context information providers, and applications or services need to access them to get context information in a homogeneous way. In this way we require homogeneous context service providers' interfaces. Independently developed services and applications must understand context meanings or semantics to advertise themselves to potential users, so we require a formal way to represent context. High-level context information, such as "the user is in a garden", may not be possible to obtain form context service providers, although those provide information that lets the system to infer other contextual information. Mobile users are mainly interested in information that relates to their environment. In this way we require a mechanism that finds the most suitable service to user situation from a wide mobile information service environment.

We are addressing these issues in our work in progress on a context aggregation service (Context Modeling component) (see section 5) that integrates and reasons over objects context information to maintain a 
coherent model of objects of the physical and information environment and its location relations, including users, places, coordinates, geographic areas, things and services. Our object model is represented by an object ontology that supports object representation, object classification and its location relationships, as well as context-aware service discovery for context-aware applications.

\section{A CONTEXT MODEL FOR SERVICE DISCOVERY}

Context information may be derived from elements of the physical world in which we all live, the technical environment in which a software application resides, or even a virtual or conceptual environment that does not, in reality, exist at all. In order to support such a diverse potential range of context information we have designed a flexible object-oriented model that can represent and monitor the parts of those environments that are of relevance to citizens and services. The aforementioned parks, water mains, and graffiti hotspots are examples of real world objects that are represented within our model. Conceptual objects such as complaints and complaint services are also represented within the same model space.

Our model is composed of objects. Everything in the model is an object and anything can be represented by one or more objects. Objects can represent anything from a real-world object to an abstract virtual concept: Objects can also represent the characteristics/properties and relationships of other objects.

An important concept in our nodel is that any object may be tied to a validity rule: a logical expression that may test and compare the attributes of any object within the model. An object is only visible to external observers if its validity rule evaluates to true, so objects may fade into and out of existence as the model changes over time. Additionally, as the model understands the identity of the observer (in terms of an object within the model) validity rules may be constructed such that certain objects will be valid, and therefore visible, to some observers whilst remaining invalid, and therefore invisible, to others. These observer-dependent validity rules form the backbone of our context-aware service discovery mechanism. For example, a parks complaint service may have a validity rule such that the observer must be within the park that the service is related to. In such a way an observer of the model will only ever see (discover) services relevant for their current context.

A novel approach is also being taken with regard to the representation of location: we view it purely as a relationship between two objects. We call 
this relationship the "IsIn" relationship. The "IsIn" relationship is represented as an object, like any other, with attributes that link to the objects representing the entity and the place it is located (not that any object can serve as a place). Chains of "IsIn" relationships can be built up and will typically terminate in the Earth object. The "IsIn" relationship may be parameterized with values to define its precise nature. For example, an "IsIn" relationship between a person and the Earth can be parameterized with a latitude and longitude in order to specify exactly where on Earth the person is. Manipulating different types of location data has long been established as a difficult problem (Leonhardt and Magee, 1996) but our approach offers a flexible solution to combine, compare and reason with location data from different sources and of widely different types.

\section{CONCLUSIONS AND FUTURE WORK}

In this paper we have argued that citizens should be able to access local government services directly in the time and place in which they are needed. We have presented a set of user requirements for m-government services and the derived logic architectural model for an open service-oriented architecture. We argue that a context modeling component is crucial in supporting context-aware m-government services. We have presented our work on the design of a context modeling component and its application for a report complaint service scenario. As future work several m-government services are being developed - the citizen complaint service is our particular focus - and will be trialed in ccllaborating municipalities within Portugal, Italy and Poland during 2005 .

\section{ACKNOWLEDGMENTS}

This work has been funded by the IS'T project USE-ME.GOV (IST-2002002294). We would like to thank Ricardo J. Machado, João M. Fernandes e Paula Monteiro from Universidade do Minho their contribution on the formal derivation of USE-ME.GOV platform requirements.

\section{References}

Abowd, G., Atkeson, C., Hong, J., Long, S., Kooper, R., and Pinkerton, M., 1997, Cyberguide: a mobile context-aware tour guide, Wireless Networks. 3(5):421-433.

Amold, K.. O'Sullivan, B., Scheifler, R., Waldo, J., and Wollrath, A., 1999, The Jini specification, Addison-Wesley, Reading, USA.

Bassara, A., Filipowska, A., Wisniewski, M., and Zebrowski, P., 2004, USE-ME.GOV project deliverable D5.2.4: Meta-protocol of service types, Poznan University. 
CENTURI21 consortium, 1999, The centuri21 project web site (Fev 28, 2004); http://www.centuri21.org.

Cheverst, K., Davies, N., Mitchell, K., and Friday, A, 2000, Experiences of developing and deploying a context-aware tourist guide: The GUIDE project, in: MobiCom 2000, ACM Press, Boston, pp 20-31.

Dey, A., Salber, D., Abowd, G., and Futakawa, M, 1999, The conference assistant: Combining context-awareness with wearable computing, in: 3rd International Symposium on Wearable Computers (ISWC'9), San Francisco, pp 21-28.

Government of Malta, 2003, M-government web site (May 15, 2005); http://www.mobile.gov.mt.

José, R., Pinto, H., Meneses, F., Vilas Boas, N., Rodrigues H., and Moreira, A., 2003, System support for integrated ubiquitous computing environments, in: System Support for Ubiquitous Computing Workshop at Ubicomp 2003, Seattle.

Leonhardt, U., and Magee, J., 1996, Towards a general location service for mobile environments, in: Proceedings of the 3rd IEEE Workshop on Services in Distributed and Networked Environments, Macau, pp 43-50.

Machado, R. J., Fernandes, J. M., Monteiro, P., and Rodrigues H., 2005, Transformation of UML models for service-oriented software architectures, in: ECBS 2005, IEEE Computer Society Press, Greenbelt, pp 73-82.

McGrath, R., 2000, Discovery and its discontents: discovery protocols for ubiquitous computing, Technical Report UIUCDCS-R-2000-2145, Department of Computer Science, University of Illinois at Urbana-Champaign, April.

Mobhaile project, 2004, The Mobhaile project web site (May 15, 2005); http://www.mobhaile.ie/.

OASIS, 2003, Universal Description, Discovery and Integration of business for the Web (May 15, 2005); http://www.uddi.org/.

OMG, 2004, Common Object Request Broker Architecture (CORBA/IIOP) specification (May 15, 2005); http://www.omg.org/technology/documents/formal/corba_iiop.htm.

Pandora consortium, 2001, The Pandora project web site (May 15, 2005); http://www.istpandora.org.

Salber, D., Dey, A., and Abowd, G. D., 1999, Th Context Toolkit: Aiding the development of context-enabled applications, in: $C H 1^{\prime} 99$, ACM Press, Pittsburgh, pp 15-20.

Satyanarayanan, Mi., 2001, Pervasive computing: Vision and challenges, IEEE Personal Communications, 8(4):10-17.

Schilit, B. N., Adanrs, N. I., and Want R., 1994, Context-aware computing applications, in: Workshop on Mobile Computing Systems and Applications, IEEE Computer Society, Santa Cruz, CA, pp 85-90.

Schmidt, A., Beigl, M., and Gellersen, H., 1998, There is more to context than location, in: Interactive Applications of Mobile Computing, Rostock, Germany.

USE-ME.GOV consortium, 2002, The USE-ME.GOV project (IST-2002-002294) web site (May 15, 2005); http://www.usemegov.org/.

Visual Admin consortium, 2000, The Visual Admin project (IST-2000-28248) web site (Feb 28, 2004); http://www.visual-admin.net/.

World Wide Web consortium, 2004, Owl-s 1.1 - web ontology language semantics (May 15, 2005); http://www.daml.org/services/owl-s/1.1/. 